PROCEEDINGS OF THE

AMERICAN MATHEMATICAL SOCIETY

Volume 128, Number 6, Pages 1761-1768

S 0002-9939(99)05303-4

Article electronically published on October 27, 1999

\title{
ON THE BOUNDARY OF ATTRACTORS WITH NON-VOID INTERIOR
}

\author{
KA-SING LAU AND YOU XU
}

(Communicated by David R. Larson)

\begin{abstract}
Let $\left\{f_{i}\right\}_{i=1}^{N}$ be a family of $N$ contractive mappings on $\mathbb{R}^{d}$ such that the attractor $K$ has nonvoid interior. We show that if the $f_{i}$ 's are injective, have non-vanishing Jacobian on $K$, and $f_{i}(K) \cap f_{j}(K)$ have zero Lebesgue measure for $i \neq j$, then the boundary $\partial K$ of $K$ has measure zero. In addition if the $f_{i}$ 's are affine maps, then the conclusion can be strengthened to $\operatorname{dim}_{H}(\partial K)<d$. These improve a result of Lagarias and Wang on self-affine tiles.
\end{abstract}

\section{INTRODUCTION}

A function $f: \mathbb{R}^{d} \rightarrow \mathbb{R}^{d}$ is called a contraction if $\|f(\mathbf{x})-f(\mathbf{y})\| \leq r\|\mathbf{x}-\mathbf{y}\|$ for all $\mathbf{x}, \mathbf{y} \in \mathbb{R}^{d}$, where $r<1$ is a constant. If equality holds, then $f$ is called a similarity and $r$ is called the contractive ratio of $f$. Let $\left\{f_{i}\right\}_{i=1}^{N}$ be a family of contractions on $\mathbb{R}^{d}$ and let $K$ be the corresponding attractor. The Hausdorff dimension and the $\alpha$-dimensional Hausdorff measure of $K$ are denoted by $\operatorname{dim}_{H}(K)$ and $\mathcal{H}^{\alpha}(K)$ respectively. We say that $\left\{f_{i}\right\}_{i=1}^{N}$ satisfies the open set condition (OSC) if there exists an open set $O$ such that $\bigcup_{i=1}^{N} f_{i}(O) \subset O$ and $f_{i}(O) \cap f_{j}(O)=\emptyset$ for $i \neq j$. It is well-known that if the contractions $f_{i}$ are all similarities, then OSC implies that the Hausdorff dimension of $K$ equals the similarity dimension $\alpha$ which is the unique number determined by $\sum_{i=1}^{N} r_{i}^{\alpha}=1$. The work of Bandt and Graf [2] and Schief [12] showed that OSC is equivalent to $\mathcal{H}^{\alpha}(K)>0$. If $\alpha=d$, the condition is further equivalent to the interior $K^{\circ} \neq \emptyset$.

Let $\mathbb{M}_{d}(\mathbb{R})$ denote the class of real $d \times d$ matrices and let $\mu$ denote the Lebesgue measure on $\mathbb{R}^{d}$. A matrix $B$ is called expanding if all of its eigenvalues satisfy $\left|\lambda_{i}\right|>1$. Let $\left\{\mathbf{a}_{1}, \mathbf{a}_{2}, \cdots, \mathbf{a}_{N}\right\} \subset \mathbb{R}^{d}$ and let $f_{i}(\mathbf{x})=B^{-1} \mathbf{x}+\mathbf{a}_{i}, \mathbf{x} \in \mathbb{R}^{d}$, be the affine transformations. Then under an appropriate metric on $\mathbb{R}^{d}$, the $f_{i}$ 's are all contractions [10. It follows that the attractor $K$ exists 8. If in addition $|\operatorname{det} B|=N$, then $\mu(K)>0$ is equivalent to $K^{\circ} \neq \emptyset$. In this case $K$ is called a self-affine tile. Lagarias and Wang [10] proved that the boundary $\partial K$ of such $K$

Received by the editors January 8, 1998 and, in revised form, July 23, 1998.

2000 Mathematics Subject Classification. Primary 28A80, 52C22; Secondary 28A78.

Key words and phrases. Boundary, Hausdorff dimension, self-affine tiles, self-similarity, singular values.

The first author was partially supported by the RGC grant CUHK4057/98P. 
has Lebesgue measure zero. In this note we will consider a few extensions of this result. We show that for a very general class of self-affine attractors in $\mathbb{R}^{d}$, the Hausdorff dimension of the boundary is strictly less than $d$.

For a function $f: \mathbb{R}^{d} \rightarrow \mathbb{R}^{d}$ and a compact set $E \subset \mathbb{R}^{d}$, we say that $f \in C^{1}(E)$ if $f$ has continuous first order partial derivatives on a neighborhood of $E$. The Jacobi determinant of $f$ at $\mathbf{x}$ is denoted by $J_{f}(\mathbf{x})$.

Theorem 1.1. Let $\left\{f_{i}\right\}_{i=1}^{N}$ be a family of contractions on $\mathbb{R}^{d}$ and $K$ the corresponding attractor with non-void interior. Suppose $f_{i} \in C^{1}(K), 1 \leq i \leq N$, are injective on $K$ with $J_{f_{i}}(\mathbf{x}) \neq 0$, and $\mu\left(f_{i}(K) \cap f_{j}(K)\right)=0$ when $i \neq j$. Then $\mu(\partial K)=0$.

It is easy to verify that the condition $\mu\left(f_{i}(K) \cap f_{j}(K)\right)=0$ is equivalent to

$$
\sum_{i=1}^{N} \mu\left(f_{i}(K)\right)=\mu(K), \text { or } \int \sum_{K}^{N}\left|J_{f_{i}}(\mathbf{x})\right| d \mathbf{x}=\mu(K) .
$$

One sufficient condition for the above equalities to hold is $\sum_{i=1}^{N}\left|J_{f_{i}}(\mathbf{x})\right|=1$ on all points of $K$. In particular when $f_{i}(\mathbf{x})=A_{i} \mathbf{x}+\mathbf{a}_{i}, A_{i} \in \mathbb{M}_{d}(\mathbb{R})$, are affine contractions, then the condition reduces to $\sum_{i=1}^{N}\left|\operatorname{det} A_{i}\right|=1$. In this case, we prove the following stronger result.

Theorem 1.2. Let $f_{i}(\mathbf{x})=A_{i} \mathbf{x}+\mathbf{a}_{i}, 1 \leq i \leq N$, be a family of affine contractions on $\mathbb{R}^{d}$ and let $K$ be the corresponding attractor. If $K^{\circ} \neq \emptyset$ and $\sum_{i=1}^{N}\left|\operatorname{det} A_{i}\right|=1$, then $\operatorname{dim}_{H}(\partial K)<d$.

Note that in the theorem we allow some $A_{i}$ 's to be singular. Using this theorem, we prove the following corollaries.

Corollary 1.3. Let $\left\{f_{i}\right\}_{i=1}^{N}$ be a family of contractive similarities on $\mathbb{R}^{d}$ and $K$ the corresponding attractor. If $K^{\circ} \neq \emptyset$ and the similarity dimension of $\left\{f_{i}\right\}_{i=1}^{N}$ is $d$, then $\operatorname{dim}_{H}(\partial K)<d$.

Corollary 1.4. Let $\left\{\mathbf{a}_{1}, \mathbf{a}_{2}, \cdots, \mathbf{a}_{N}\right\} \subset \mathbb{R}^{d}$ and let $B \in \mathbb{M}_{d}(\mathbb{R})$ be expanding with $|\operatorname{det} B|=N$. If the attractor $K$ of $\left\{f_{i}: f_{i}(\mathbf{x})=B^{-1} \mathbf{x}+\mathbf{a}_{i}\right\}_{i=1}^{N}$ has non-void interior, then $\operatorname{dim}_{H}(\partial K)<d$.

The second corollary improves Lagarias and Wang's result on the boundary of self-affine tiles. As mentioned before, the condition in the above two corollaries that $K$ has non-void interior is equivalent to the condition that $K$ has positive Lebesgue measure. We point out that it was proved recently $[9]$ that the Hausdorff dimension of the boundary of a self-affine tile in $\mathbb{R}^{d}$ can be arbitrarily close to $d$.

Note that in 11 Bandt also considered the rotations and reflections of a tile. We call a finite group $\mathbb{W}$ of matrices with determinant \pm 1 a symmetry group of an expanding matrix $B$ if $B \mathbb{W}=\mathbb{W} B$. Let $w_{i} \in \mathbb{W}, \mathbf{a}_{i} \in \mathbb{R}^{d}$ and $f_{i}(\mathbf{x})=w_{i} B^{-1} \mathbf{x}+\mathbf{a}_{i}$, $i=1, \cdots, N$. Then $\left\{f_{i}\right\}_{i=1}^{N}$ can generate more exotic tiles such as Levy's curve, Heighway dragon, etc. [1]. Theorem 1.2 also applies to these tiles. 
Corollary 1.5. Let $B \in \mathbb{M}_{d}(\mathbb{R})$ be expanding with $|\operatorname{det} B|=N$ and $\mathbb{W}$ be a symmetry group of $B$. Let $f_{i}(\mathbf{x})=w_{i} B^{-1} \mathbf{x}+\mathbf{a}_{i}, w_{i} \in \mathbb{W}, \mathbf{a}_{i} \in \mathbb{R}^{d}, i=1, \cdots, N$. If the attractor $K$ of $\left\{f_{i}\right\}_{i=1}^{N}$ has non-void interior, then $\operatorname{dim}_{H}(\partial K)<d$.

We remark that if $K$ is the attractor generated by affine transformations consisting of one single matrix, then there are simple criteria to determine that $K$ has non-void interior [10. However very little is known if there is more than one matrix (see [1 for the case in Corollary 1.5). The estimation of the dimension of the boundary is far from being understood. It seems that only for a few particular classes of self-similar tiles (7], 4] and [13) has the exact dimension been calculated.

For an attractor $K$ of similarities $\left\{f_{i}\right\}_{i=1}^{N}$ in $\mathbb{R}^{d}$ with similarity dimension $\alpha$, it is well-known that if $\left\{f_{i}\right\}_{i=1}^{N}$ satisfies OSC, then $\mathcal{H}^{\alpha}\left(f_{i}(K) \cap f_{j}(K)\right)=0$ for $i \neq j$ [5, Corollary 8.7]. Our theorem sharpens this result.

Theorem 1.6. Let $\left\{f_{i}\right\}_{i=1}^{N}$ be a family of contractive similarities on $\mathbb{R}^{d}$ with similarity dimension $\alpha, 0<\alpha \leq d$, and let $K$ be the corresponding self-similar set. If $\left\{f_{i}\right\}_{i=1}^{N}$ satisfies the OSC, then $\operatorname{dim}_{H}\left(f_{i}(K) \cap f_{j}(K)\right)<\alpha$ for $i \neq j$.

\section{Definitions And PReliminaries}

Let $\mathbb{N}$ be the set of natural numbers. For $\mathbb{S}=\{1,2, \cdots, N\}$, let $\mathbb{S}^{n}=\underbrace{\mathbb{S} \times \cdots \times \mathbb{S}}_{n}$ and $\mathbb{S}^{*}=\bigcup_{n \in \mathbb{N}} \mathbb{S}^{n}$. The length of $\mathbf{s}=\left(s_{1} \cdots s_{n}\right) \in \mathbb{S}^{n}$ is denoted by $|\mathbf{s}|(=n)$. If $\mathbf{i}=\left(i_{1} i_{2} \cdots i_{n_{1}}\right), \mathbf{j}=\left(j_{1} j_{2} \ldots j_{n_{2}}\right)$, then we define

$$
\mathbf{i j}=\left(i_{1} i_{2} \cdots i_{n_{1}} j_{1} j_{2} \cdots j_{n_{2}}\right) \text {. }
$$

For a subset $E \subset \mathbb{R}^{d}$, its diameter is defined as $|E|=\sup \{|\mathbf{x}-\mathbf{y}|: \mathbf{x}, \mathbf{y} \in E\}$. For $f_{i}: \mathbb{R}^{d} \rightarrow \mathbb{R}^{d}, i \in \mathbb{S}$, we define $E_{\mathbf{s}}=f_{\mathbf{s}}(E)=f_{s_{1}} \circ f_{s_{2}} \circ \cdots \circ f_{s_{n}}(E)$. It is easy to see that if all $f_{i}$ 's are contractive, then $f_{\mathbf{s}}$ is also contractive and its contractive ratio is $r_{\mathbf{s}}=r_{s_{1}} r_{s_{2}} \cdots r_{s_{n}}$.

We use $B_{a}(\mathbf{x})$ to denote the closed ball with center $\mathbf{x}$ and radius $a$. Let $\|\cdot\|$ denote the Euclidean norm on $\mathbb{R}^{d}$ and the norm of a matrix $A \in \mathbb{M}_{d}(\mathbb{R})$ is

$$
\|A\|=\max \left\{\frac{\|A \mathbf{x}\|}{\|\mathbf{x}\|}: \mathbf{x} \in \mathbb{R}^{d},\|\mathbf{x}\| \neq 0\right\} .
$$

The spectral radius of $A$ is $\lambda_{\max }=\max _{1 \leq i \leq d}\left|\lambda_{i}\right|$, where $\lambda_{i}$ are the eigenvalues of $A$. If $Q$ is the closed unit ball in $\mathbb{R}^{d}$ and $f(\mathbf{x})=A \mathbf{x}+\mathbf{a}$ is an affine mapping with $A$ non-singular, then $A(Q)$ is an ellipsoid. The lengths of the principle semi-axes of $A(Q)$ are singular values $\sigma_{1} \geq \sigma_{2} \geq \cdots \geq \sigma_{d}>0$ of $A$. These singular values are also the positive roots of the eigenvalues of $A^{T} A$, where $A^{T}$ is the transpose of $A$. The norm and the singular values of $A$ have the following relationships:

$$
\begin{gathered}
\sigma_{1}=\max \left\{\frac{\|A \mathbf{x}\|}{\|\mathbf{x}\|}:\|\mathbf{x}\|=1\right\}=\|A\|, \\
\sigma_{d}=\min \left\{\frac{\|A \mathbf{x}\|}{\|\mathbf{x}\|}:\|\mathbf{x}\| \neq 0\right\}=\min \left\{\frac{\|\mathbf{y}\|}{\left\|A^{-1} \mathbf{y}\right\|}:\|\mathbf{y}\| \neq 0\right\}=1 /\left\|A^{-1}\right\| .
\end{gathered}
$$

Also we have

$$
|\operatorname{det} A|=\sqrt{\operatorname{det}\left(A^{T} A\right)}=\sigma_{1} \sigma_{2} \cdots \sigma_{d}
$$


Using the spectral radius formula $\lambda_{\max }=\lim _{n \rightarrow \infty}\left\|A^{n}\right\|^{1 / n}$ [11, Theorem 10.13], it is easy to see the following:

Lemma 2.1. Let $A \in \mathbb{M}_{d}(\mathbb{R})$ with spectral radius $\lambda_{\max }$ and $\rho>\lambda_{\max }$. Then there exists a constant $c>0$ depending on $A$ such that for any $n \in \mathbb{N},\left\|A^{n}\right\| \leq c \cdot \rho^{n}$.

\section{Proofs of the Results}

Proof of Theorem 1.1. We know that for any $n \in \mathbb{N}$,

$$
K=\bigcup_{i=1}^{N} f_{i}(K)=\bigcup_{\mathbf{s} \in \mathbb{S}^{n}} f_{\mathbf{s}}(K)=\bigcup_{\mathbf{s} \in \mathbb{S}^{n}} K_{\mathbf{s}}
$$

Since $f_{i}$ are contractive, $\max _{\mathbf{s} \in \mathbb{S}^{n}}\left|K_{\mathbf{s}}\right| \rightarrow 0$ when $n \rightarrow \infty$. We can find $\varepsilon>0, \mathbf{x}_{0} \in K$ and $\mathbf{k} \in \mathbb{S}^{m}$ for some large $m$ such that

$$
K_{\mathbf{k}} \subset B_{\varepsilon}\left(\mathbf{x}_{0}\right) \subset K^{\circ} \neq \emptyset .
$$

Since $J_{f_{i}}(\mathbf{x}) \neq 0$ and $f_{i}$ are injective for all $i=1, \cdots, N$, it is easy to see that for any $\mathbf{s} \in \mathbb{S}^{n}, f_{\mathbf{s}}$ is a homeomorphism between $K$ and $f_{\mathbf{s}}(K)$. It follows that $f_{\mathbf{s}} \in C^{1}(K), f_{\mathbf{s}}^{-1} \in C^{1}\left(K_{\mathbf{s}}\right), K_{\mathbf{s}}^{\circ}=f_{\mathbf{s}}\left(K^{\circ}\right)$ and $\partial K_{\mathbf{s}}=f_{\mathbf{s}}(\partial K)$. We claim that if $\mathbf{i}, \mathbf{j} \in \mathbb{S}^{m}$ and $\mathbf{i} \neq \mathbf{j}$, then $\mu\left(K_{\mathbf{i}} \cap K_{\mathbf{j}}\right)=0$. Indeed, suppose $\mathbf{i}=\left(i_{1} \cdots i_{m}\right)$ and $\mathbf{j}=\left(j_{1} \cdots j_{m}\right)$. Let $l$ be the smallest integer such that $i_{l} \neq j_{l}$. Then

$$
\begin{aligned}
\mu\left(K_{\mathbf{i}} \cap K_{\mathbf{j}}\right) & =\mu\left(f_{i_{1} \cdots i_{l-1}}\left(K_{i_{l} \cdots i_{m}}\right) \cap f_{i_{1} \cdots i_{l-1}}\left(K_{j_{l} \cdots j_{m}}\right)\right) \\
& =\mu\left(f_{i_{1} \cdots i_{l-1}}\left(K_{i_{l} \cdots i_{m}} \cap K_{j_{l} \cdots j_{m}}\right)\right) \leq \mu\left(f_{i_{1} \cdots i_{l-1}}\left(K_{i_{l}} \cap K_{j_{l}}\right)\right) \\
& =\int_{K_{i_{l}} \cap K_{j_{l}}}\left|J_{f_{i_{1} \cdots i_{l-1}}}(\mathbf{x})\right| d \mathbf{x}=0,
\end{aligned}
$$

where the second equality holds because $f_{i_{1} \cdots i_{l-1}}$ is injective, and the last equality holds because $\mu\left(K_{i_{l}} \cap K_{j_{l}}\right)=0$. This proves the claim.

Now since $\partial K_{\mathbf{k}} \subset K_{\mathbf{k}} \subset K^{\circ}$, for $\mathbf{x} \in \partial K_{\mathbf{k}}$, we can find a sequence $\left\{\mathbf{y}_{i}\right\}_{i=1}^{\infty}$ such that $\mathbf{y}_{i} \rightarrow \mathbf{x}$ and $\mathbf{y}_{i} \in K^{\circ} \backslash K_{\mathbf{k}}$. It follows that $\mathbf{y}_{i} \in K_{\mathbf{t}_{i}} \backslash K_{\mathbf{k}}$ for some $\mathbf{t}_{i} \in \mathbb{S}^{m}$. Since there are only finitely many elements in $\mathbb{S}^{m}$, we can assume, by passing to subsequence, that $\mathbf{y}_{i} \in K_{\mathbf{t}}$ for a fixed $\mathbf{t} \in \mathbb{S}^{m}$. Since $\mathbf{x}$ is the limit point of the sequence and $K_{\mathbf{t}}$ is compact, we have $\mathbf{x} \in K_{\mathbf{t}}$. This is true for any $\mathbf{x} \in \partial K_{\mathbf{k}}$. Therefore $\partial K_{\mathbf{k}} \subset K_{\mathbf{k}} \cap\left(\bigcup_{\mathbf{t} \in \mathbb{S}^{m}, \mathbf{t} \neq \mathbf{k}} K_{\mathbf{t}}\right)$ and hence

$$
\mu\left(\partial K_{\mathbf{k}}\right) \leq \mu\left(K_{\mathbf{k}} \cap\left(\bigcup_{\mathbf{t} \in \mathbb{S}^{m}, \mathbf{t} \neq \mathbf{k}} K_{\mathbf{t}}\right)\right) \leq \sum_{\mathbf{t} \in \mathbb{S}^{m}, \mathbf{t} \neq \mathbf{k}} \mu\left(K_{\mathbf{k}} \cap K_{\mathbf{t}}\right)=0
$$

by the claim. Note that $\partial K=f_{\mathbf{k}}^{-1}\left(\partial K_{\mathbf{k}}\right)$; we have

$$
\mu(\partial K)=\int_{\partial K_{\mathbf{k}}}\left|J_{f_{\mathbf{k}}^{-1}}(\mathbf{x})\right| d \mathbf{x}=0 .
$$


Proof of Theorem 1.2. For any $n \in \mathbb{N}$, we have

$$
K=\bigcup_{i=1}^{N} f_{i}(K)=\bigcup_{\mathbf{s} \in \mathbb{S}^{n}} f_{\mathbf{s}}(K)=\bigcup_{\mathbf{s} \in \mathbb{S}^{n}} K_{\mathbf{s}}
$$

where

$$
f_{\mathbf{s}}(\mathbf{x})=A_{s_{1}} \cdots A_{s_{n}} \mathbf{x}+A_{s_{1}} \cdots A_{s_{n-1}} \mathbf{a}_{s_{n}}+\cdots+A_{s_{1}} \mathbf{a}_{s_{2}}+\mathbf{a}_{s_{1}} .
$$

We use $A_{\mathbf{s}}$ to denote $A_{s_{1}} \cdots A_{s_{n}}$. Since $f_{i}$ 's are all contractions, $\left\|A_{i}\right\|<1$ for $i=$ $1,2, \cdots, N$. Let $\rho=\max _{1<i<N}\left\{\left\|A_{i}\right\|\right\}, \kappa=|K|$. Then $\rho<1$ and $\left|K_{\mathbf{s}}\right| \leq\left\|A_{\mathbf{s}}\right\| \kappa \leq \rho^{n} \kappa$. Since $K^{\circ} \neq \emptyset$, there is an open ball $B_{\varepsilon}\left(\mathbf{x}_{0}\right) \subset K^{\circ}$. Pick an integer $m$ big enough so that $\rho^{m} \kappa<\frac{\varepsilon}{2}$. We have $B_{\varepsilon / 2}\left(\mathbf{x}_{0}\right) \subset \bigcup_{\mathbf{s} \in \mathbb{S}^{m}} K_{\mathbf{s}}$. If $A_{\mathbf{s}}$ is singular, then $K_{\mathbf{s}}$ has volume zero. So there exists a $\mathbf{k} \in \mathbb{S}^{m}$ such that $A_{\mathbf{k}}$ is non-singular and $K_{\mathbf{k}} \cap B_{\varepsilon / 2}\left(\mathbf{x}_{0}\right) \neq \emptyset$. It follows that $K_{\mathbf{k}} \subset B_{\varepsilon}\left(\mathbf{x}_{0}\right) \subset K^{\circ}$.

Now without loss of generality, we suppose $A_{1}, \cdots, A_{M}, 0 \leq M<N$, are singular matrices and $A_{M+1}, \cdots, A_{N}$ are non-singular. Set

$$
\widetilde{\mathbb{S}^{*}}=\left\{\mathbf{s}: \mathbf{s} \in \mathbb{S}^{*}, s_{i} \notin\{1,2, \cdots, M\}, i=1, \cdots,|\mathbf{s}|\right\} .
$$

Then for any $\mathbf{s} \in \widetilde{\mathbb{S}^{*}}, A_{\mathbf{s}}$ is non-singular, and for any $\mathbf{s} \in \mathbb{S}^{*} \backslash \widetilde{\mathbb{S}^{*}}, A_{\mathbf{s}}$ is singular. Let $\mathbf{j} \in \widetilde{\mathbb{S}^{*}}$. Then

$$
K_{\mathbf{j k}}=f_{\mathbf{j k}}(K)=f_{\mathbf{j}}\left(K_{\mathbf{k}}\right) \subset f_{\mathbf{j}}\left(K^{\circ}\right)=\left(f_{\mathbf{j}}(K)\right)^{\circ} \subset K^{\circ} .
$$

Let $E=K_{\mathbf{k}} \cup\left(\bigcup_{\mathbf{j} \in \mathbb{S}^{*}} K_{\mathbf{j k}}\right)$ and $F=K \backslash E$. Then $E \subset K^{\circ}$ and

$$
\partial K=K \backslash K^{\circ} \subset K \backslash E=F .
$$

For $l \in \mathbb{N}$ and $\mathbf{s} \in \mathbb{S}^{m l}$, we write $\mathbf{s}=\left(\mathbf{s}_{1}, \cdots, \mathbf{s}_{l}\right) \in \mathbb{S}^{m} \times \cdots \times \mathbb{S}^{m}=\mathbb{S}^{m l}$. Let

$$
J=\left\{\mathbf{s}: \mathbf{s} \in\left(\mathbb{S}^{m l} \cap \widetilde{\mathbb{S}^{*}}\right), \mathbf{s}_{j}=\mathbf{k} \text { for some } j\right\} \text { and } L=\mathbb{S}^{m l} \backslash J .
$$

Then $K=\left(\bigcup_{\mathbf{s} \in J} K_{\mathbf{s}}\right) \cup\left(\bigcup_{\mathbf{s} \in L} K_{\mathbf{s}}\right)$ and

$$
F=K \backslash E \subset K \backslash\left(\bigcup_{\mathbf{s} \in J} K_{\mathbf{s}}\right) \subset \bigcup_{\mathbf{s} \in L} K_{\mathbf{s}} .
$$

If we let $U$ be a ball of radius $a>0$ and contain $K$, then

$$
\partial K \subset F \subset \bigcup_{\mathbf{s} \in L} K_{\mathbf{s}} \subset \bigcup_{\mathbf{s} \in L} U_{\mathbf{s}}
$$

From $\sum_{i=1}^{N}\left|\operatorname{det} A_{i}\right|=1$, it is easy to show that $\sum_{\mathbf{s} \in L}\left|\operatorname{det} A_{\mathbf{s}}\right|=\left(1-\left|\operatorname{det} A_{\mathbf{k}}\right|\right)^{l}$. We will use the identity later.

For each non-singular $A_{\mathbf{s}}$, we use an idea from Falconer [6, p132] to get an estimate. We know that $U_{\mathbf{s}}$ is an ellipsoid with principal semi-axes $a \sigma_{1}(\mathbf{s}) \geq a \sigma_{2}(\mathbf{s}) \geq$ $\cdots \geq a \sigma_{d}(\mathbf{s})>0$, where $\sigma_{i}(\mathbf{s})(1 \leq i \leq d)$ are the singular values of $A_{\mathbf{s}}$. The ellipsoid is contained in a rectangular parallelepiped $P$ of side lengths $2 a \sigma_{1}$ (s), $2 a \sigma_{2}(\mathbf{s})$, 
$\cdots, 2 a \sigma_{d}(\mathbf{s})$. We may cover $P$ by at most $\gamma$ cubes of side $2 a \sigma_{d}(\mathbf{s})$, where

$$
\gamma=\prod_{i=1}^{d-1} \frac{4 a \sigma_{i}(\mathbf{s})}{2 a \sigma_{d}(\mathbf{s})}=2^{d-1}\left(\prod_{i=1}^{d} \sigma_{i}(\mathbf{s})\right)\left(\sigma_{d}(\mathbf{s})\right)^{-d}=2^{d-1}\left|\operatorname{det} A_{\mathbf{s}}\right|\left(\sigma_{d}(\mathbf{s})\right)^{-d} .
$$

The diameter of each cube is $2 a \sigma_{d}(\mathbf{s}) \sqrt{d}$. Note that $\sigma_{d}(\mathbf{s}) \leq \sigma_{1}(\mathbf{s})=\left\|A_{\mathbf{s}}\right\| \leq \rho^{m l}$. Let $\delta=2 a \rho^{m l} \sqrt{d}$. Then

$$
\mathcal{H}_{\delta}^{\beta}\left(U_{\mathbf{s}}\right) \leq \gamma\left(2 a \sigma_{d}(\mathbf{s}) \sqrt{d}\right)^{\beta}=c_{\beta}\left|\operatorname{det} A_{\mathbf{s}}\right|\left(\sigma_{d}(\mathbf{s})\right)^{\beta-d},
$$

where $c_{\beta}=2^{d-1}(2 a \sqrt{d})^{\beta}$ is a constant depending on $\beta$.

For each singular matrix $A_{\mathbf{s}}, U_{\mathbf{s}}$ is contained in a hyperplane. So if $\beta>d-1$, then $\mathcal{H}^{\beta}\left(U_{\mathbf{s}}\right)=0$ and hence $\mathcal{H}_{\delta}^{\beta}\left(U_{\mathbf{s}}\right)=0$ for any $\delta>0$. Let $\widetilde{L}=L \cap \widetilde{\mathbb{S}^{*}}$. Then for every $\mathbf{s} \in L \backslash \widetilde{L}, A_{\mathbf{s}}$ is singular, and hence for $\beta>d-1$,

$$
\mathcal{H}_{\delta}^{\beta}(F) \leq \mathcal{H}_{\delta}^{\beta}\left(\bigcup_{\mathbf{s} \in L} U_{\mathbf{s}}\right)=\mathcal{H}_{\delta}^{\beta}\left(\bigcup_{\mathbf{s} \in \widetilde{L}} U_{\mathbf{s}}\right) \leq \sum_{\mathbf{s} \in \widetilde{L}} c_{\beta}\left|\operatorname{det} A_{\mathbf{s}}\right|\left(\sigma_{d}(\mathbf{s})\right)^{\beta-d} .
$$

Since $\sigma_{d}(\mathbf{s})$ is the smallest singular value of $A_{\mathbf{s}}$,

$$
\sigma_{d}(\mathbf{s})=\frac{1}{\left\|\left(A_{\mathbf{s}}\right)^{-1}\right\|} \geq \frac{1}{\left\|A_{s_{m l}}^{-1}\right\| \cdots\left\|A_{s_{1}}^{-1}\right\|}=\tau_{M+1}^{n_{1}} \cdots \tau_{N}^{n_{N-M}}
$$

where $n_{1}+n_{2}+\cdots+n_{N-M}=m l$ and $\tau_{i}=1 /\left\|A_{i}^{-1}\right\|$ is the smallest singular value of $A_{i}, M+1 \leq i \leq N$. It is clear that $0<\tau_{i} \leq\left\|A_{i}\right\|<1$. Let $\tau=$ $\min \left\{\tau_{i}: i=M+1, \cdots, N\right\}$; then $\sigma_{d}(\mathbf{s}) \geq \tau^{m l}$. So for $d-1<\beta<d$,

$$
\begin{aligned}
\mathcal{H}_{\delta}^{\beta}(F) & \leq \sum_{\mathbf{s} \in \tilde{L}} c_{\beta}\left|\operatorname{det} A_{\mathbf{s}}\right| \tau^{m l(\beta-d)}=c_{\beta} \tau^{m l(\beta-d)} \sum_{\mathbf{s} \in \widetilde{L}}\left|\operatorname{det} A_{\mathbf{s}}\right| \\
& =c_{\beta} \tau^{m l(\beta-d)} \sum_{\mathbf{s} \in L}\left|\operatorname{det} A_{\mathbf{s}}\right|=c_{\beta}\left(\tau^{m(\beta-d)}\left(1-\left|\operatorname{det} A_{\mathbf{k}}\right|\right)\right)^{l} .
\end{aligned}
$$

Let $\tau^{m(\beta-d)}\left(1-\left|\operatorname{det} A_{\mathbf{k}}\right|\right)=1$, i.e.,

$$
\beta=d-\frac{\ln \left(1-\left|\operatorname{det} A_{\mathbf{k}}\right|\right)}{m \ln \tau}<d .
$$

Then $\mathcal{H}_{\delta}^{\beta}(F)<c_{\beta}$. Let $l \rightarrow \infty$; then $\delta \rightarrow 0$ and $\mathcal{H}^{\beta}(F) \leq c_{\beta}$. It follows that $\operatorname{dim}_{H}(F) \leq \beta<d$. Since $\partial K \subset F$, we conclude that $\operatorname{dim}_{H}(\partial K)<d$.

Proof of Corollary 1.3. Let $f_{i}(\mathbf{x})=A_{i} \mathbf{x}+\mathbf{a}_{i}$ be the similarities and let $r_{i}$ be the contractive ratios. Then $\left|\operatorname{det} A_{i}\right|=r_{i}^{d}$. It follows from the assumption that $\sum_{i=1}^{N}\left|\operatorname{det} A_{i}\right|=\sum_{i=1}^{N} r_{i}^{d}=1$ and Theorem 1.2 applies.

Proof of Corollary 1.4. Let $A=B^{-1}$. Then $f_{i}(\mathbf{x})=A \mathbf{x}+\mathbf{a}_{i}, i=1, \cdots, N$. For any $n \in \mathbb{N}, K=\bigcup_{\mathbf{s} \in \mathbb{S}^{n}} f_{\mathbf{s}}(K)$ and

$$
f_{\mathbf{s}}(\mathbf{x})=A^{n} \mathbf{x}+A^{n-1} \mathbf{a}_{s_{n}}+\cdots+A \mathbf{a}_{s_{2}}+\mathbf{a}_{s_{1}} .
$$

Suppose the eigenvalues of $B$ are $\lambda_{i}, 1 \leq i \leq d$, with $\left|\lambda_{1}\right| \geq \cdots \geq\left|\lambda_{d}\right|>r>1$. Then the eigenvalues of $A^{n}$ are $\lambda_{i}^{-n}, 1 \leq i \leq d$. Using Lemma 2.1 we can find $n$ large enough so that $\left\|A^{n}\right\| \leq c \cdot r^{-n}<1$ for some constant $c>0$ independent of 
$n$. It follows that for each $\mathbf{s} \in \mathbb{S}^{n}, f_{\mathbf{s}}$ is an affine contraction with corresponding $\operatorname{matrix} A^{|\mathbf{s}|}=A^{n}$. Note that $\sum_{\mathbf{s} \in \mathbb{S}^{n}}\left|\operatorname{det} A^{|\mathbf{s}|}\right|=N^{n} \cdot N^{-n}=1$ and hence Theorem 1.2 implies the corollary immediately

Proof of Corollary 1.5. Let $A=B^{-1}$. Then $f_{i}(\mathbf{x})=w_{i} A \mathbf{x}+\mathbf{a}_{i}, \mathbf{x} \in \mathbb{R}^{d}, i=$ $1, \cdots, N$. For any $n \in \mathbb{N}$, we have $K=\bigcup_{\mathbf{s} \in \mathbb{S}^{n}} f_{\mathbf{s}}(K)$, where

$$
f_{\mathbf{s}}(\mathbf{x})=w_{s_{1}} A \cdot w_{s_{2}} A \cdots w_{s_{n}} A \mathbf{x}+w_{s_{1}} A \cdots w_{s_{n-1}} A \mathbf{a}_{s_{n}} \cdots+w_{s_{1}} A \mathbf{a}_{s_{2}}+\mathbf{a}_{s_{1}} .
$$

By $B \mathbb{W}=\mathbb{W} B$, it is easy to see that for any $w_{i} \in \mathbb{W}, A w_{i}=w_{j} A$ for some $w_{j} \in \mathbb{W}$. Hence $w_{s_{1}} A \cdot w_{s_{2}} A \cdots w_{s_{n}} A=w A^{n}$ for some $w \in \mathbb{W}$. It follows that $\left\|w_{s_{1}} A \cdot w_{s_{2}} A \cdots w_{s_{n}} A\right\| \leq\left(\max _{w \in \mathbb{W}}\|w\|\right)\left\|A^{n}\right\|$. Now the rest is the same as in the proof of Corollary 1.4.

Proof of Theorem 1.6. To prove this theorem, we need to use a result in 12 and a similar proof as in Theorem 1.2. For $E \subset \mathbb{R}^{d}$ and $\varepsilon>0$, let

$$
U(\varepsilon, E)=\{\mathbf{x}:|\mathbf{x}-\mathbf{y}|<\varepsilon \text { for some } \mathbf{y} \in E\} .
$$

For $\mathbf{s} \in \mathbb{S}^{*}$, define $G_{\mathbf{s}}=U\left(\varepsilon r_{\mathbf{s}}, K_{\mathbf{s}}\right)$. Then from [12] we know that there exists a $\mathbf{k} \in \mathbb{S}^{*}$ such that for some small $\varepsilon>0$, the set $O=G_{\mathbf{k}} \cup\left(\bigcup_{\mathbf{j} \in \mathbb{S}^{*}} G_{\mathbf{j k}}\right)$ is an OSC set for $\left\{f_{i}\right\}_{i=1}^{N}$. Suppose $|\mathbf{k}|=m$. Let $E=K \cap O$ and $F=K \backslash E$. For $l \in \mathbb{N}$, let

$$
J=\left\{\mathbf{s}=\left(\mathbf{s}_{1}, \cdots, \mathbf{s}_{j}, \cdots, \mathbf{s}_{l}\right) \in \mathbb{S}^{m l}: \mathbf{s}_{j}=\mathbf{k} \text { for some } j\right\} \text { and } L=\mathbb{S}^{m l} \backslash J
$$

as in the proof of Theorem 1.2. Then

$$
F=K \backslash O \subset K \backslash \bigcup_{\mathbf{j} \in J} G_{\mathbf{j}} \subset K \backslash \bigcup_{\mathbf{j} \in J} K_{\mathbf{j}} \subset \bigcup_{\mathbf{j} \in L} K_{\mathbf{j}} .
$$

Since $\sum_{i=1}^{N} r^{\alpha}=1$, it is easy to see that $\sum_{\mathbf{s} \in L} r_{\mathbf{s}}^{\alpha}=\left(1-r_{\mathbf{k}}^{\alpha}\right)^{l}$. Let $\widetilde{r}=\max _{1 \leq i \leq N}\left\{r_{i}\right\}$, $r=\min _{1 \leq i \leq N}\left\{r_{i}\right\}$ and $\kappa=|K|$. Then we have, for $\mathbf{s} \in L, r^{l m} \kappa \leq\left|K_{\mathbf{s}}\right|=r_{\mathbf{s}} \kappa \leq \widetilde{r}^{l m} \kappa$. Let $\delta_{l}=\widetilde{r}^{m} \kappa$. Then for $0<\beta<\alpha$,

$$
\mathcal{H}_{\delta_{l}}^{\beta}(F) \leq \mathcal{H}_{\delta_{l}}^{\beta}\left(\bigcup_{\mathbf{j} \in L} K_{\mathbf{j}}\right) \leq \sum_{\mathbf{s} \in L}\left|K_{\mathbf{s}}\right|^{\beta}=\sum_{\mathbf{s} \in L}\left(r_{\mathbf{s}} \kappa\right)^{\beta} \leq \kappa^{\beta}\left(r^{m(\beta-\alpha)}\left(1-r_{\mathbf{k}}^{\alpha}\right)\right)^{l} .
$$

Hence by the similar arguments as in the proof of Theorem 1.2, we have $\operatorname{dim}_{H}(F) \leq$ $\beta<\alpha$ if

$$
\beta=\alpha-\frac{\ln \left(1-r_{\mathbf{k}}^{\alpha}\right)}{m \ln r} .
$$

Now suppose $i \neq j$. Since $K=E \cup F$, it is clear that

$$
f_{i}(K) \cap f_{j}(K) \subset\left(f_{i}(E) \cap f_{j}(E)\right) \cup f_{i}(F) \cup f_{j}(F) .
$$

But $O$ is an OSC set and $E \subset O$, so $f_{i}(E) \cap f_{j}(E) \subset f_{i}(O) \cap f_{j}(O)=\emptyset$. Hence $f_{i}(K) \cap f_{j}(K) \subset f_{i}(F) \cup f_{j}(F)$. Since $f_{i}$ and $f_{j}$ are similarities, $\operatorname{dim}_{H}\left(f_{i}(F)\right)=$ $\operatorname{dim}_{H}\left(f_{j}(F)\right)=\operatorname{dim}_{H}(F)<\alpha$. Therefore $\operatorname{dim}_{H}\left(f_{i}(K) \cap f_{j}(K)\right) \leq \operatorname{dim}_{H}(F)<$ $\alpha$. 


\section{ACKNOWLEDGMENT}

We would like to thank Professor D. J. Hebert for many helpful discussions.

\section{REFERENCES}

[1] C. Bandt, Self-similar sets 5. Integer matrices and fractal tilings of $\mathbb{R}^{n}$, Proc. Amer. Math. Soc. 112(1991), 549-562. MR 92d:58093

[2] C. Bandt and S. Graf, Self-similar sets 7. A characterization of self-similar fractals with positive Hausdorff measure, Proc. Amer. Math. Soc.114(1992), 995-1001. MR 93d:28014

[3] F. M. Dekking, Recurrent sets, Adv. Math. 44(1982), 78-104. [MR 84e:52023]

[4] P. Duvall and J. Keesling, The Hausdorff dimension of the boundary of the Levy dragon, preprint 1998.

[5] K. J. Falconer, "The Geometry of Fractal Sets", Cambridge University Press, Cambridge, 1985. MR 88d:28001

[6] K. J. Falconer, "Fractal Geometry: Mathematical Foundation and Applications", Wiley, New York, 1990. MR 92j:28008

[7] W. J. Gilbert, The fractal dimension of sets derived from complex bases, Canad. Math. Bull. 29(1986), 495-500. MR 88b:28014

[8] J. E. Hutchinson, Fractals and self-similarity, Indiana Univ. Math. J. 30(1981), 713-747. MR 82h:49026

[9] R. Kenyon, J. Li, R. Strichartz, and Y. Wang, Geometry of self-affine tiles II, preprint 1998.

[10] J. C. Lagarias and Y. Wang, Self-affine Tiles in $\mathbb{R}^{n}$, Adv. Math. 121(1996), 21-49. MR 97d:52034

[11] W. Rudin, "Functional Analysis", McGraw-Hill, Inc. New York, 1991. MR 92k:46001

[12] A. Schief, Separation properties for self-similar sets, Proc. Amer. Math. Soc. 122(1994),111115. MR 94k:28012

[13] R. Strichartz and Y. Wang, Geometry of self-affine tiles I, preprint 1998.

Department of Mathematics, The Chinese University of Hong Kong, Hong Kong

E-mail address: kslau@math.cuhk.edu.hk

Department of Mathematics, University of Pittsburgh, Pittsburgh, Pennsylvania 15260

E-mail address: yoxst+@pitt.edu 